\title{
A STUDENT-DESIGNED LABORATORY COMPANION BOARD FOR A COURSE ON MICROPROCESSOR INTERFACING AND EMBEDDED SYSTEMS
}

\author{
David King, Brendan Montgomery, Matt Sippert, and Naraig Manjikian* \\ *Department of Electrical and Computer Engineering, Queen's University, Kingston, Ontario, Canada K7L 3N6 \\ naraig.manjikian@queensu.ca
}

\begin{abstract}
This paper describes the production and usage of a student-designed laboratory companion board for a core course on microprocessor interfacing and embedded systems in the Department of Electrical and Computer Engineering at Queen's University. The companion board connects to existing digital-only hardware in order to provide analog-digital conversion and other features. The design and prototype implementation of the companion board was pursued as a capstone design project by three students during the 2014-2015 academic session. The student designers had agreed from the outset to allow their intellectual property to be used for educational purposes. In mid-2015, a production run of 30 boards was completed. A total of more than 300 students then used the companion board in laboratory activity during the Fall 2015 and Fall 2016 terms. The introduction of this student-designed equipment into the laboratory has been a positive development that provides an example to students of how material learned in multiple courses can be integrated together to produce a valuable outcome.
\end{abstract}

Keywords: Microprocessor interfacing, analog-digital conversion, embedded systems, laboratory hardware, capstone design projects.

\section{INTRODUCTION}

This paper presents the design and implementation of a laboratory companion board, and describes the experience from two years of using this board in the core course ELEC 371 Microprocessor Interfacing and Embedded Systems in the Department of Electrical and Computer Engineering at Queen's University. The companion board connects to existing equipment in the laboratory and provides features not available in the existing equipment.

The detailed design and prototype implementation of the companion board was undertaken by three students (D. King., B. Montgomery, and M. Sippert) as their capstone undergraduate engineering design project during the 2014-2015 academic session [1], under the supervision of a faculty member (N. Manjikian) who has also served as the sole instructor for the relevant ELEC 371 course for many years. The student designers of the companion board graciously agreed at the outset to allow their intellectual property to be used for educational purposes. Once the project had been completed by the students, the supervising faculty member oversaw the fabrication of 30 production boards in mid-2015. Software developed by the student designers during their project activity for the purpose of demonstrating the operation of the companion board was also essential for testing the production boards and ensuring their readiness for laboratory use.

To incorporate the companion board into laboratory activity for ELEC 371, the supervising faculty member (N. Manjikian) made adjustments in the selection of topics pursued in laboratory exercises. During the Fall 2015 and Fall 2016 terms, a total of more than 300 students used the companion board. Careful management of the new equipment ensured that no hardware problems were encountered. Overall, the introduction of the new equipment can be characterized as a positive development.

The remainder of this paper briefly outlines some related work, describes the laboratory environment and technical aspects for the development of the companion board to serve in that environment, summarizes the usage of the production boards in laboratory activity, offers some reflections on the experience, and concludes with some final comments.

\section{SAMPLES OF RELATED WORK}

The most direct inspiration for the project in this paper is the description by Manjikian and Simmons [2] of an effort in 1998 to enhance the same ELEC 371 course for this paper using companion hardware for a commercial board with the 8-bit $68 \mathrm{HC} 11$ microcontroller [3] that was being used at that time for laboratory activity. In fact, an initial companion board for the $68 \mathrm{HC} 11$ hardware was developed by Simmons for ELEC 371 even earlier in 1992. Thus, there is a history of using companion hardware for ELEC 371, and the effort described in this paper is a logical continuation, but the primary difference with past efforts is the student-driven nature of this effort. 
A related example of a larger and more ambitious nature is described by Nooshabadi and Garside [4]. A multiyear collaborative project from the early 2000s involving a total of three universities produced two custom boards. The first board included an ARM microcontroller and Xilinx programmable logic chips, and the second board for expansion provided an LCD module, switches, LEDs, and additional external connections. Thus, the expansion board from this example of prior work is equivalent to a companion board as discussed in this paper.

\section{LABORATORY ENVIRONMENT AND DEVELOPMENT OF COMPANION BOARD}

This section provides an overview of the laboratory environment and equipment, a technical summary of the companion board, and a description of the development and production process.

\subsection{Laboratory Setting}

Undergraduate laboratory activities for courses in the Department of Electrical and Computer Engineering are pursued in Beamish-Munro Hall, which serves all engineering programs at Queen's University. The building provides two laboratory plazas with stations for computers and other equipment. Figure 1 depicts the smaller plaza with approximately 25 stations. Figure 2 depicts the larger plaza with approximately 40 stations and a sliding partition to divide the space in half.

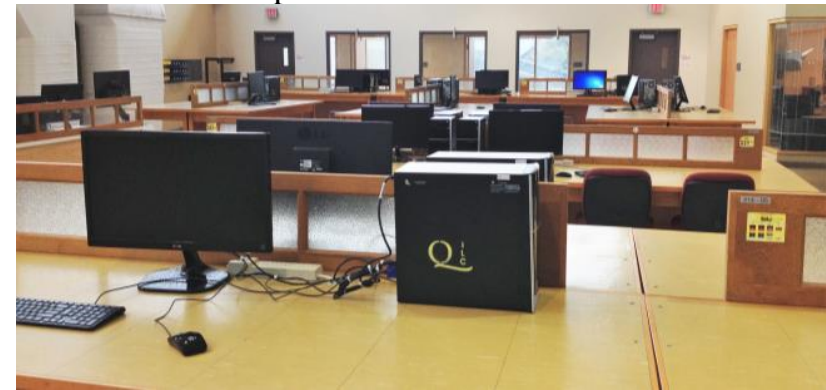

Fig. 1. Smaller laboratory plaza in Beamish-Munro Hall.

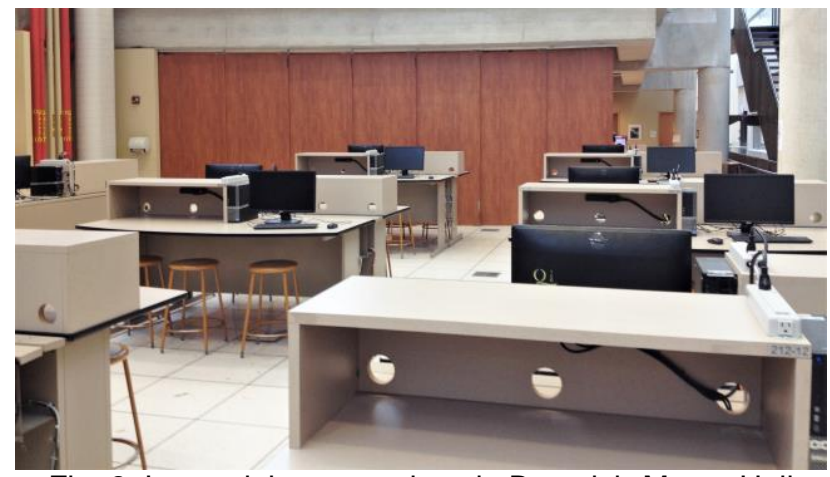

Fig. 2. Larger laboratory plaza in Beamish-Munro Hall with sliding partition in place to divide space in half.

\subsection{Existing Equipment}

Figure 3 depicts the Altera DE0 board [5] in use since the Fall 2011 term in laboratory activity for courses in digital logic, computer organization, and microprocessor/embedded systems. It is a digital-only platform that was selected for its low unit cost and its reliance on a single USB cable for both power and communication. The board contains an Altera programmable logic chip. For laboratory activity, students configure their own digital circuits generated with the Quartus II software tool [6]. Students can also configure vendor-provided embedded computer systems based on the Altera Nios II 32-bit processor [7], and use the Monitor Program software tool [8] on an attached desktop computer for interacting with the embedded computer system in the DE0 board. At Queen's University, the boards are maintained in their original cardboard boxes and stored conveniently in cabinets within the two laboratory plazas of Beamish-Munro Hall.

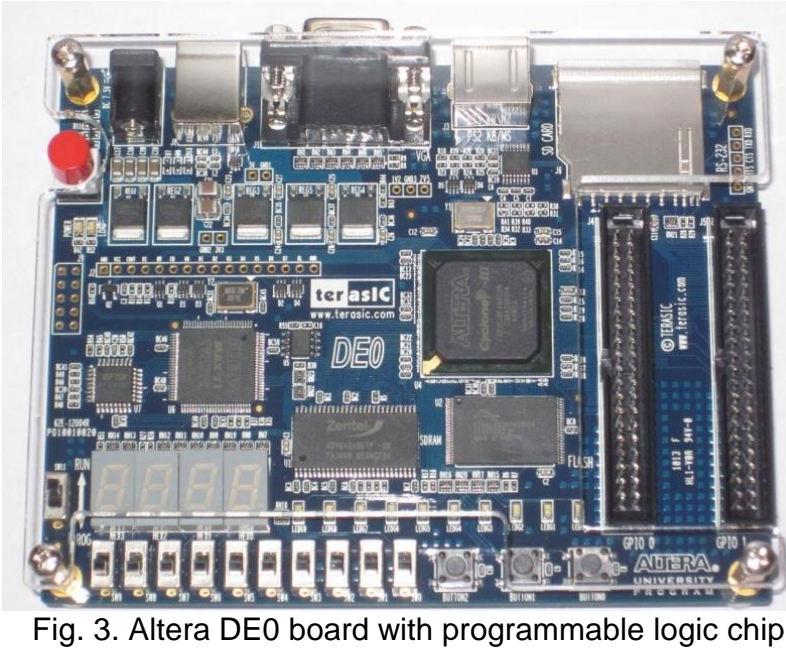
and expansion headers on the right-hand side.

In Fig. 3, the right-hand side of the DE0 board has two 40-pin expansion headers that connect directly to the programmable logic chip. The vendor-provided computer systems include parallel port interfaces that allow software to use the expansion headers for interaction with appropriately designed external hardware. The companion board that is described in this paper uses one of the expansion headers for connection to the DE0 board.

\subsection{Summary of Features for Companion Board}

The primary features of the companion board are for analog-digital interfacing in order to address the fact that the DE0 board is limited to digital interfacing capabilities. A ribbon cable connects the companion board to one of the 40-pin expansion headers on the DE0 board, and the companion board derives its power through this cable. 
The companion board has three single-channel analog-todigital conversion chips and three 4-to-1 analog multiplexer chips to support a total of 12 analog inputs. Several inputs are hardwired to built-in features on the companion board such as an accelerometer module, a potentiometer, and a photoresistor. The companion board also has one digital-to-analog conversion chip with four analog output channels. One output channel is hardwired to a variablebrightness LED on the companion board.

The companion board also has a three-pin header for a standard servomotor and a two-pin header for a battery pack with four AA batteries dedicated for the servomotor. The pulse-width-modulated (PWM) signal for the servomotor is one of the 40 pins connecting the companion board and a DE0 board. The PWM signal can be generated by software executed by the processor embedded in the programmable chip to change the pin output according to intervals dictated by timer interrupts. Alternatively, the PWM signal output can be generated directly by custom logic using dedicated counter circuits to dictate the time intervals, all incorporated into a custom input/output interface added to the vendor-provided computer system.

\subsection{Development Process in Capstone Project}

Capstone projects in the Department of Electrical and Computer Engineering are defined in the Winter term of the third year. Students either select from supervisorproposed topics or convince a supervisor to oversee a student-proposed effort. Then, students submit an initial project proposal document. During the Fall and Winter terms of the fourth year, students pursue design, implementation, and testing activities with a \$400 departmentprovided budget, submitting a more detailed technical proposal document at the outset, and a final report at the end. All projects are demonstrated at an annual openhouse event in Beamish-Munro Hall during the Winter term. At the open house, a panel of external judges assesses the project demonstrations to award prizes.

For the capstone project that ultimately produced the companion board, the student designers were given general guidance from the supervising faculty member to develop analog-digital interfacing features as a minimum requirement. The possible addition of other features such as servomotor support was left primarily to the consideration of the students. The students made specific choices for built-in elements such as an accelerometer module.

After establishing the board features, the students prepared an initial schematic design, and acquired the necessary electronic and other components. Figure 4 shows the breadboard prototype of the companion board prepared by the students, which was tested with a DE0 board.

A two-layer printed-circuit board with dimensions of approximately $9.1 \mathrm{~cm} \times 10 \mathrm{~cm}$ was designed by the stu- dents using the EAGLE software tool, and the output was sent to AP Circuits in Alberta for fabrication. Using a duplicate set of components, the students completed the final assembly and soldering. Figure 5 depicts the result.

After board fabrication, a minor problem was discovered in the routing of one of connections to a chip socket. This problem was resolved by using on-site fabrication facilities in Beamish-Munro Hall to produce a small board with the necessary correction to mount in the affected socket. The small correction board is visible at the bottom-left of Fig. 5, next to the ribbon cable connector.

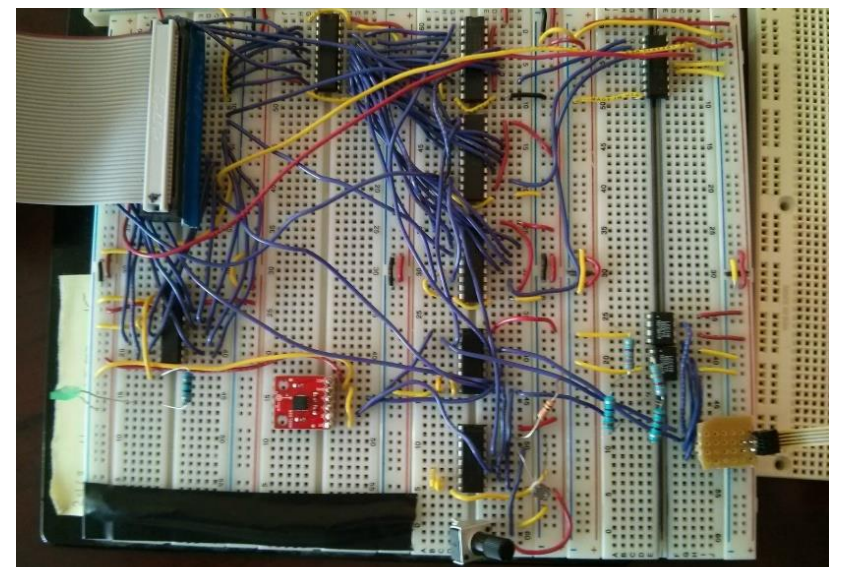

Fig. 4. Breadboard prototype of companion board.

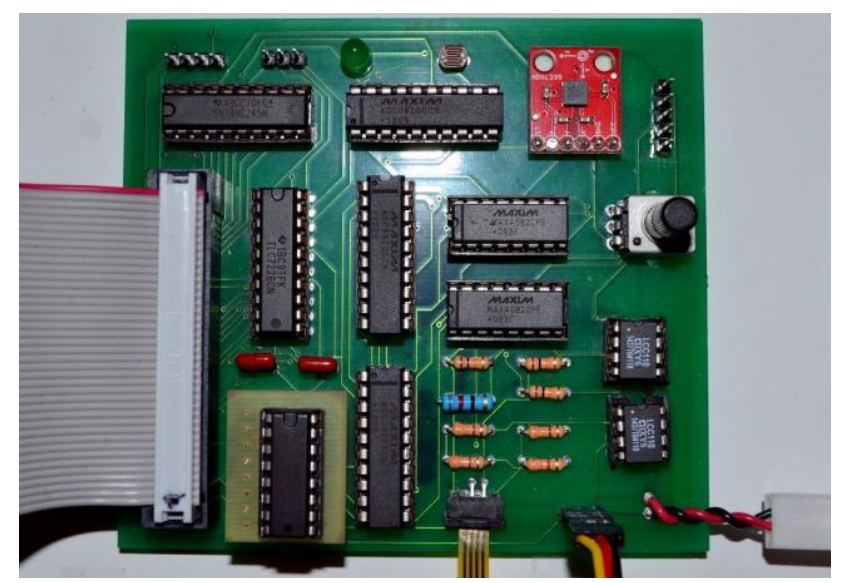

Fig. 5. Initial printed-circuit board implementation with small board (bottom-left) to resolve a connection problem.

Finally, the students prepared a custom software program for the Nios II processor in the embedded computer system in the programmable logic chip of the DE0 board to interact with the companion board hardware through the parallel port interface for one of the 40-pin expansion headers. This program utilized the graphical capabilities of the vendor-provided embedded computer system for the DE0 board. Figure 6 depicts how this software displays the digital conversions of analog input values as bar graphs. The students used this program for their openhouse demonstration of their completed capstone project. 


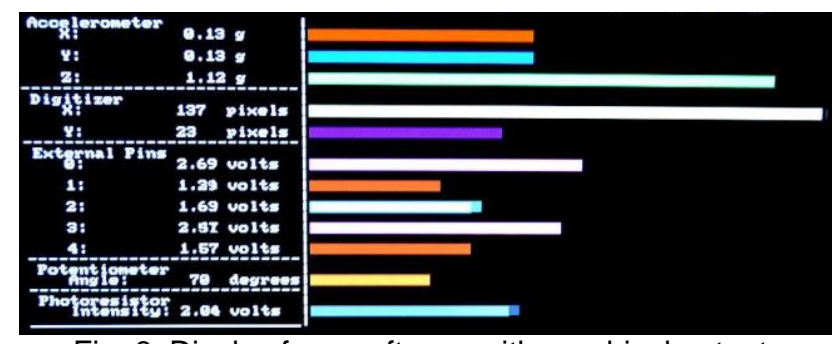

Fig. 6. Display from software with graphical output.

\subsection{Final Production of Boards for Laboratory}

In January 2015, following successful implementation and testing of the breadboard prototype by the student designers, a proposal for production financing was submitted by the supervising faculty member to the Better Equipment Donation fund of the undergraduate student Engineering Society. The proposal was approved, and funding for 30 production boards was provided.

At the end of the Winter 2015 term, after the students had completed not only their capstone project but also their entire undergraduate degree program, the final printed-circuit board specifications with all necessary revisions were provided by the students to the supervising faculty member as the last deliverable related to the project.

In April-May 2015, all necessary parts were purchased, and the production run of 30 printed-circuit boards was fabricated by AP Circuits in Alberta. Figure 7 shows the fabricated boards received in early June 2015. Final assembly and soldering was completed by technical staff at Queen's University by mid-July 2015 in preparation for the Fall 2015 term. Figure 8 shows the storage bin containing the completed boards; the numbers on the bags reflect the same serial numbers marked on the boards for managing the collection of production boards. This storage bin is kept for convenience in the same laboratory cabinet space as the Altera DE0 boards.

Thorough testing of the 30 production boards was performed by the supervising faculty member using the student-developed graphical software that was shown in Fig. 6. The availability of this software was vital to the success of the overall effort. The testing process led to a surprising discovery: $10 \%$ of the ribbon cables were defective, which was unexpected for such a simple low-cost component. Granted, the selected cables were less expensive ones without strain relief, but the defect rate was still somewhat surprising. The testing also revealed just a single missed solder connection on a single board across the entire collection of 30 boards. The combination of conscientious design, implementation, and testing efforts of the students during their 2014-2015 capstone project, coupled with the care and attention by technical staff during final assembly ensured a successful outcome.

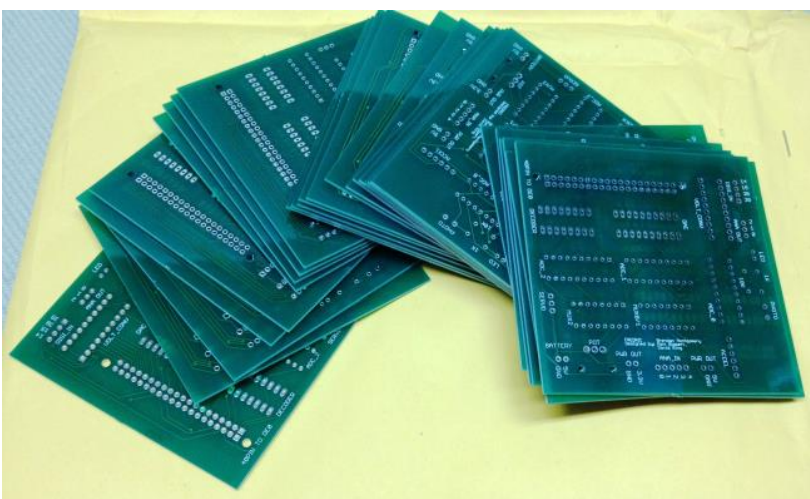

Fig. 7. Blank printed-circuit boards.

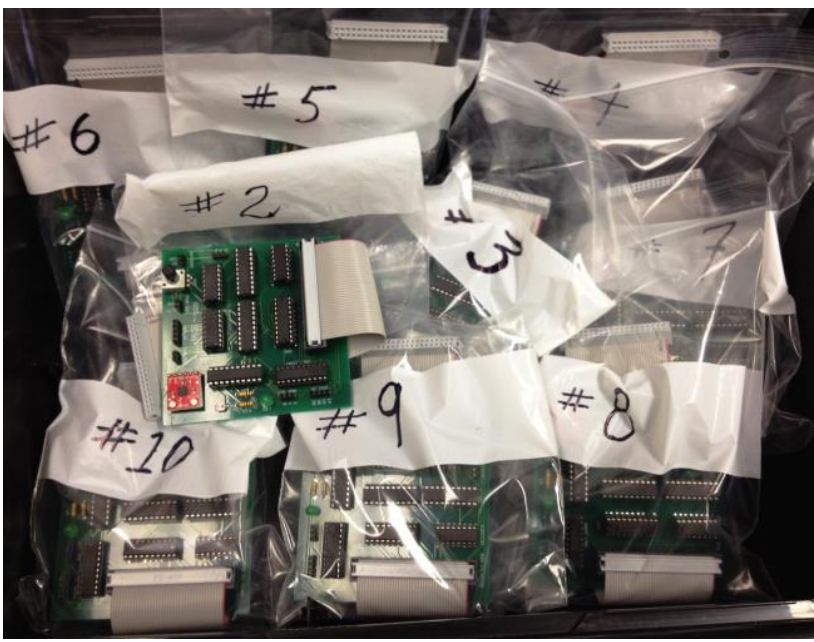

Fig. 8. Completed boards in storage bin.

\section{USAGE OF COMPANON BOARD}

This section summarizes the first two years of using the production companion boards in ELEC 371. First, various constraints related to the sequencing of laboratory topics are outlined, and then the actual activities for analogdigital interfacing in the laboratory are described.

\subsection{Constraints of Laboratory Sequencing}

For over two decades, ELEC 371 laboratory activity consisted of weekly two-hour sessions for all students in the course, even during periods of high enrollment such as in the late 1990s and early 2000s with more than 200 students, then again from the early 2010s after a decade of reduced enrolment. Prior to 2012 when the 8-bit $68 \mathrm{HC} 11$ microcontroller was the platform for the course, the sequence of topics for laboratory activity was established and refined by Simmons and Manjikian [2] to cover programming for input/output ports, timers, and interrupts. In 2012, the platform was changed by Manjikian as part of a 
broader multi-course enhancement effort. The new platform became the 32-bit Nios II processor configured in a programmable logic chip. Similar laboratory topics related to ports, timers, and interrupts were pursued with appropriate adaptations to reflect the new platform.

Beginning in 2015, the introduction of the companion board required changes to the sequencing of laboratory topics in the second half of the term (the fundamental laboratory aspects in the first half could not be compromised). A single two-hour laboratory session with the new hardware was deemed insufficient to acquire familiarity and also pursue a technically meaningful experience. Therefore, two of the twelve weekly sessions were devoted to analog-digital interfacing with the companion board. With this new activity being inserted, other activity required removal. It was decided that students would no longer create their own embedded computer systems based on the Nios II processor, an effort that had been pursued in the two laboratory sessions at the end of the term in the 2013 and 2014 offerings of the course.

In 2016, additional challenges emerged when the laboratory activity in ELEC 371 was altered to have less frequent bi-weekly sessions with longer three-hour duration, resulting in a net reduction of $25 \%$ in laboratory time for each student compared to previous years. The primary administrative reason was a desire to reduce pressure arising from higher demand for the shared laboratory plazas in Beamish-Munro Hall from many courses in different engineering programs. A more specific consideration from the perspective of the ELEC 371 instructor was an observed decline in student preparation for weekly activity and reduced student attention/diligence during two-hour weekly laboratory sessions, likely due to workload pressures in other courses during the same term. The change in laboratory timetabling for 2016 required further adjustments to reduce and consolidate activity from successive two-hour sessions into a single three-hour session. Nonetheless, the use of the companion board was retained in two successive bi-weekly sessions.

\subsection{Analog-Digital Interfacing Activities}

To support laboratory activity with the companion board, the supervising faculty member as instructor prepared a six-page technical document based on the implementation details. This document names the student designers of the companion board as co-authors in recognition of their effort. The document describes bit assignments for the expansion parallel port and other operational requirements for communicating between the embedded computer system within the programmable logic chip on the DE0 board and the companion board. For software, students are also provided with a partially completed source file that provides a template for using the analog- to-digital and digital-to-analog conversion features of the companion board. As preparation for the first of two laboratory sessions involving the companion board, students must study the technical documentation and complete the missing portions of the provided source file.

In the first laboratory session with the companion board, the activity for students involves preparing software to control LED displays on the DE0 board based on the digital conversion of the analog input from the companion board potentiometer. Then, the converted analog input is used as the digital input for conversion to analog output to vary the brightness of the LED on the companion board.

The second laboratory session with the companion board enlarges the scope of utilized features to encompass the analog inputs from the accelerometer module. Students are required to obtain converted digital values from the accelerometer analog inputs and use that data in a more complex software program involving timer interrupts. The program must use the accelerometer input to repeatedly update the cursor position of text output to an attached desktop computer from the computer system embedded within the logic chip of the DE0 board. By physically tilting the companion board on two axes, the horizontal and vertical position of the on-screen cursor for printed text is modified.

The servomotor connection of the companion board has not yet been used in the laboratory due in part to scheduling constraints and the other topics that must be covered in laboratory exercises. There is also the added cost and logistical overhead of providing a collection of servomotors and battery packs to pursue servomotorbased activity during laboratory sessions. The servomotor feature has, however, been used by the supervising faculty member for demonstration purposes during ELEC 371 lectures, and also in lectures for a second-year course on digital logic.

\section{REFLECTIONS ON EXPERIENCE}

First and foremost, the student designers and the supervising faculty member agree that the entire project for the development of the companion board was a positive experience that produced a valuable outcome. For the student designers, the careful approach and good planning throughout the project reduced stress and contributed to success. For the supervising faculty member, there was a reduced need to intervene and to advise the project group. With only one significant prototyping issue involving a connection problem that was resolved in a straightforward manner, the essential analog-digital conversion features of interest were successfully implemented.

For the production boards, the assistance of technical staff was valuable in acquiring the components, fabricating the printed-circuit boards, and completing the final 
assembly. There was only one minor issue with initially ordering an incorrect accelerometer module, but because that module was actually the one needed in a different course, there was, in the end, no problem. There was also only one missed solder connection in the final assembly for the entire production run of 30 boards.

Finally, for using the companion boards in the laboratory in Fall 2015 and then again in Fall 2016, there were no significant hardware problems due to careful management of the companion boards. For the two laboratory sessions where the companion boards were involved, the two boards were connected by teaching assistants and the instructor in advance and left in that state by the students to avoid any further cable issues. Students generally treated the boards with care as directed by the instructor. The template-based approach for students preparing software to use the companion board ensured that the vast majority of students were able to obtain learning benefits from working with analog-digital conversion.

The companion board provides a valuable ability to have students engage in analog-digital interfacing within the established digital-only environment based on the $\mathrm{Al}-$ tera DE0 board. This benefit is certainly important. From the perspective of the supervising faculty member, however, a more important message to students with their exposure to the companion board is that the material which is taught in several courses - electric circuits, electronics, digital logic, computer organization, and embedded systems - can be combined and applied effectively to achieve a positive and valuable result. By emphasizing the fact that a group of students pursuing the same courses was entirely responsible for the design of the hardware now being used by other students in ELEC 371, the supervising faculty member as the course instructor believes that the appreciation of the material by students can be enhanced.

\section{CONCLUSION}

The companion board project described in this paper is an example of a successful effort involving students applying knowledge acquired across multiple courses to produce a useful outcome. Building on past experience and benefits of companion hardware for laboratory use, this effort enabled the digital-only environment based on the Altera DE0 board to be extended to include analog input/output capabilities. This effort provides a model for similar projects in the future that could involve students making a contribution towards laboratory teaching.

\section{Acknowledgements}

Financial support for producing the companion boards in 2015 and initially acquiring the Altera DE0 boards in 2011 was provided by the Better Equipment Donation Fund of the undergraduate student Engineering Society of Queen's University. The authors also acknowledge the valuable contributions of Roy Campsall for final assembly and soldering of production companion boards, Steve Humphrey for coordinating printed-circuit-board fabrication, and Gary Neff for assisting in procurement of parts.

\section{References}

[1] D. King, B. Montgomery, and M. Sippert, Companion Board for Altera DEx, ELEC 490/498 capstone project final report, Dept. of Electrical and Computer Engineering, Queen's University, March 2015.

[2] N. Manjikian and S. Simmons, "Evolution and enhancements of a microprocessor systems course," IEEE Transactions on Education, Vol. 42, No. 4, November 1999. Appears in special CD-ROM supplement with article summary on p. 360.

[3] Freescale Semiconductor, M68HC11 Reference Manual, document M68HC11RM/D, revision 6.1, 2007.

[4] S. Nooshabadi and J. Garside, "Teaching embedded systems design - an international collaborative project," Proceedings of the $35^{\text {th }}$ ASEE/IEEE Frontiers in Education Conference, Indianapolis, IN, October 2005, pp. F2D26F2D30.

[5] Terasic Inc., DEO User Manual, document version 1.6, May 2012.

[6] Altera Corp., Quartus II Handbook Version 13.0 - Volume I: Design and Synthesis, document QII6V1-13.0.0, May 2013.

[7] Altera Corp., Basic Computer System for the Altera DEO Board, document version for Quartus II 13.0, May 2013.

[8] Altera Corp., Altera Monitor Program Tutorial, document version for Quartus II 13.0, May 2013. 